\title{
A review on some results on local conservation laws for certain evolution equations ${ }^{1}$
}

I.L. Freire, CMCC - Centro de Matemática, Computação e Cognição, UFABC Universidade Federal do ABC, Rua Santa Adélia, 166, Bairro Bangu, 09.210-170 Santo André, SP, Brasil

J.C.S. Sampaio, IMECC - Departamento de Matemática Aplicada, UNICAMP Universidade Estadual de Campinas, Rua Sérgio Buarque de Holanda, 651, Cidade Universitária "Zeferino Vaz", Barão Geraldo, 13083-859 Campinas, SP, Brasil.

Abstract. In this work we revisit some recent results on conservation laws for a class of fifth-order evolution equations up to fifth-order.

Key-words. Ibragimov's theorem, conservation laws, fifth-order KdV equations.

\section{Introduction}

This work corresponds to the talk [6], given by J. C. S. Sampaio, during the XXXIV CNMAC - XXXIV Congresso Nacional de Matemática Aplicada e Computacional - September, 17-21 2012, Águas de Lindóia-SP, Brasil, where it was presented and discussed some recent results [5] with respect to how to obtain conservation laws for an equation without a classical Lagrangian.

It is well known that for equations arising from the Euler-Lagrange equations, the celebrated Noether theorem provides an elegant way for finding conserved quantities for such equation, see $[1,17,20]$. However, when it is considered equations without variational structure, it is impossible to apply Noether's approach. Then the following question arises: how can one find a conserved vector for an equation without Lagrangians?

Across the last century, many approaches have been proposed in order to overcame this problem. The interested reader is directed to the reference [19], where some of these developments were discussed. In this paper we use the most recent approach proposed in [12], where it was shown a general result connecting symmetries and conservation laws, in order to find conserved vectors for the general equation

$$
u_{t}+\alpha u u_{x}+\beta u^{2} u_{x}+\gamma u_{x x x}+\mu u_{x x x x x}=0 .
$$

\footnotetext{
${ }^{1}$ This work was supported by FAPESP, grants no 2011/23538-0 and 2011/19089-6.

${ }^{2}$ igor.freire@ufabc.edu.br and igor.leite.freire@gmail.com.

3 juliocesar.santossampaio@gmail.com.
} 
Following [2], we would like to call this new formulation for finding conserved vectors as Ibragimov's theorem on conservation laws.

In this paper it is considered the equation (1.1) with the following restrictions: $(\alpha, \beta) \neq(0,0)$ and $(\gamma, \mu) \neq(0,0)$. In fact, in the case whenever $(\gamma, \mu)=(0,0)$ it is obtained a particular case of the inviscid Burgers equation, which was considered in $[2,4]$. Whenever $\alpha=\beta=0$ it is obtained a linear equation and here we are interested in nonlinear phenomena.

Apart from the previous mentioned equations, equation (1.1) includes

- The celebrated KdV equation

$$
u_{t}+\alpha u u_{x}+\gamma u_{x x x}=0 ;
$$

- General Kawahara equation

$$
u_{t}+\alpha u u_{x}+\gamma u_{x x x}+\mu u_{x x x x x}=0
$$

- Simplified modified Kawahara equation

$$
u_{t}+\beta u^{2} u_{x}+\mu u_{x x x x x}=0 ;
$$

- Simplified Kawahara equation

$$
u_{t}+\alpha u u_{x}+\mu u_{x x x x x}=0
$$

and

- Gardner equation

$$
u_{t}+6\left(u+u^{2}\right) u_{x}+u_{x x x}=0 .
$$

All of these equations is coming from the celebrated $\mathrm{KdV}$ equation, which was deduced by Korteweg and his student de-Vries for modeling shallow wave equations. These equations are employed in Mathematical Physics in order to describe a lot of dispersive phenomena, such as plasma phenomena, while equations of the type (1.2) are also used to model the Great Red Spot of Jupiter, see [21].

Although our main purpose is to revisit some of our previous results given in $[5,21]$, in the present work we also present some new conservation laws for equations of the class (1.1). Namely, the new conserved vectors derived by using the developments $[12,15,16]$ are

$$
\begin{aligned}
& C^{0}=u^{2}, \\
& C^{1}=\frac{2}{3} \alpha u^{3}+2 \mu u u_{x x x x}-2 \mu u_{x} u_{x x x}+\mu u_{x x}^{2},
\end{aligned}
$$

for the simplified Kawahara equation (1.5), and

$$
\begin{aligned}
& C^{0}=-u-u^{2}, \\
& C^{1}=3 u^{4}+6 u^{3}+3 u^{2}+2 u u_{x x}+u_{x x}-u_{x}^{2},
\end{aligned}
$$


for the Gardner equation (1.6).

The remaining of this paper is as the follows. In the next section we revisit the Ibragimov's theorem on conservation and the necessary tools to employ it for finding local conserved vectors. Next, in the section 3 , we show that the class of equations (1.1) is nonlinearly self-adjoint. This allows us to find local conservation laws for these equations, which will be done in the following sections. Some parts of this review closely follow our references [5, 21].

\section{Ibragimov's theory}

In this section we revisit the basic tools about the recent developments started with the fruitful work [12], where Ibragimov proved a new conservation theorem. Next we introduce the recent concept of nonlinear self-adjointness, proposed by Ibragimov in $[15,16]$.

This field, nowadays, is a rich branch in the field of group analysis and it has been attracting the attention of a big number of researchers. Many of them are interested in finding nonlinearly self-adjoint properties of equations, as it can be seen in $[2,3,4,5,7,8,9,10,11,13,14,22]$ and references therein.

\subsection{Adjoint equations and nonlinear self-adjointness}

Let $x=\left(x^{1}, \cdots, x^{n}\right)$ be $n$ independent variables, $u=u(x)$ be a dependent variable. The set of $k$ th order derivatives of $u$ is denoted by $u_{(k)}$, where $k$ in a positive integer number. Consider the set of functions depending on $x, u$ and $u$ derivatives up to a finite order.

A locally analytic function of a finite number of the variables $x, u$ and $u$ derivatives is called a differential function. The highest order of derivatives appearing in the differential function is called the order of this function. The vector space of all differential functions of finite order is denoted by $\mathcal{A}$.

The formal sum

$$
\frac{\delta}{\delta u}=\frac{\partial}{\partial u}+\sum_{j=1}^{\infty}(-1)^{j} D_{i_{1}} \cdots D_{i_{j}} \frac{\partial}{\partial u_{i_{1} \cdots i_{j}}}
$$

is the well known Euler-Lagrange operator.

Let $F \in \mathcal{A}$ be a differential function. From this function we can obtain a differential equation

$$
F\left(x, u, \cdots, u_{(s)}\right)=0
$$

and the following new differential function, called formal Lagrangian, given by $\mathcal{L}=$ $v F$, where $v=v(x)$ is another dependent variable.

Equation (2.10) is said to be nonlinearly self-adjoint if the equation obtained from the adjoint equation

$$
F^{*}\left(x, u, v, \cdots, u_{(s)}, v_{(s)}\right):=\frac{\delta \mathcal{L}}{\delta u}=0
$$


by the substitution $v=\phi(x, u)$ with a certain function $\phi(x, u) \neq 0$ is identical with the original equation (2.10), that is,

$$
\left.F^{*}\left(x, u, v, u_{(1)}, v_{(1)}, \cdots, u_{(s)}, v_{(s)}\right)\right|_{v=\phi(x, u)}=0 .
$$

Whenever (2.12) holds for a certain differential function $\phi$ such that $\phi_{u} \neq 0$ and $\phi_{x} \neq 0$, equation (2.11) is called weak self-adjoint.

In other words: equation (2.11) is said to be nonlinearly self-adjoint if there exists a function $\phi=\phi(x, u)$ such that

$$
\left.F^{*}\right|_{v=\phi}=\lambda(x, u, \cdots) F,
$$

for some differential function $\lambda=\lambda(x, u, \cdots)$.

\subsection{Ibragimov's theorem on conservation laws}

The following result was proved in [12].

Theorem 2.1 (Ibragimov's theorem on conservation laws). Let

$$
X=\xi^{i} \frac{\partial}{\partial x^{i}}+\eta \frac{\partial}{\partial u}
$$

be any symmetry (Lie point, Lie-Bäcklund, nonlocal symmetry) of equation (2.10) and (2.11) be the adjoint equation to equation (2.10). The combined system (2.10) and (2.11) has the conservation law $D_{i} C^{i}=0$, where

$$
\begin{aligned}
C^{i}= & \xi^{i} \mathcal{L}+W\left[\frac{\partial \mathcal{L}}{\partial u_{i}}-D_{j}\left(\frac{\partial \mathcal{L}}{\partial u_{i j}}\right)+D_{j} D_{k} \frac{\partial \mathcal{L}}{\partial u_{i j k}}-\cdots\right] \\
& +D_{j}(W)\left[\frac{\partial \mathcal{L}}{\partial u_{i j}}-D_{k}\left(\frac{\partial \mathcal{L}}{\partial u_{i j k}}\right)+\cdots\right] \\
& +D_{j} D_{k}(W)\left[\frac{\partial \mathcal{L}}{\partial u_{i j k}}-\cdots\right]+\cdots
\end{aligned}
$$

and $W=\eta-\xi^{i} u_{i}$.

\subsection{Algorithm}

Ibragimov's theorem on conservation laws can be resumed by the following algorithm (see $[12,2]$ for further details): given a PDE

$$
F=F\left(x, u, u_{(1)} \cdots, u_{(n)}\right)=0,
$$

- we construct a Lagrangian $\mathcal{L}=v F$.

- From the Euler-Lagrange equations, the following system is obtained:

$$
\begin{gathered}
F\left(x, u, u_{(1)} \cdots, u_{(n)}\right)=0, \\
F^{*}\left(x, u, v, \cdots, u_{(s)}, v_{(s)}\right)=0 .
\end{gathered}
$$

- We construct the conserved vector given by (2.14). 


\section{Nonlinearly self-adjoint classification of the equa- tion (1.1)}

The following theorem was proved in [5] (see also [21]).

Theorem 3.2. Equation (1.1), with $(\gamma, \mu) \neq 0$ and $(\alpha, \beta) \neq(0,0)$, is nonlinearly self-adjoint.

Remarks: Equation (1.1) is also nonlinearly self-adjoint without the requested hypothesis in the Theorem 3.2. The mentioned hypothesis only reflects the nonlinearity of the equation and the fact that we are considering dispersive equations. However, a straightforward calculation shows that we can remove them. We leave the details to the interested reader. It can also be useful, once going in this direction, to see $[2,3,4]$.

Proof. Let us denote the left side of (1.1) by

$$
F=u_{t}+\alpha u u_{x}+\gamma u_{x x x}+\mu u_{x x x x x} .
$$

Then, the adjoint equation to $F=0$ is

$$
F^{*}:=-v_{t}-\left(\alpha u+\beta u^{2}\right) v_{x}-\gamma v_{x x x}-\mu v_{x x x x x}=0 .
$$

Now we only must analyze the following two cases. Below, $a_{1}, a_{2}$ and $a_{3}$ are arbitrary constants.

- Assume $\beta=0$ in (1.1). Then the substitution

$$
\phi(x, t, u)=a_{1}(x-\alpha t u)+a_{2} u+a_{3}
$$

in (3.18) makes the adjoint equation equivalent to the original equation.

- Now suppose $\beta \neq 0$ in (1.1). Substituting $\phi(x, t, u)=a_{1} u+a_{2}$ instead of $v$ in (3.18), we obtain a multiple of (1.1).

This proves our statement.

\section{Non-local conservation laws}

Here we find nonlocal conservation laws for the equation (1.1). We follow the algorithm previously discussed in Section 2.3.

Actually, the first two steps of such a mentioned algorithm have just been done because the formal Lagrangian had already been obtained while the adjoint equation to (1.1) had also already been calculated and it is given by (3.18). 
With respect to the second step, the components of the vector are given by

$$
\begin{aligned}
C^{0}= & \tau \mathcal{L}+W v \\
C^{1}= & \xi \mathcal{L}+W\left[v\left(\alpha u+\beta u^{2}\right)+\gamma v_{x x}+\mu v_{x x x x}\right] \\
& -D_{x}(W)\left(\gamma v_{x}+\mu v_{x x x}\right)+D_{x}^{2}(W)\left(\gamma v+\mu v_{x x}\right) \\
& -D_{x}^{3}(W)\left(\mu v_{x}\right)+D_{x}^{4}(W)(\mu v),
\end{aligned}
$$

where $\mathcal{L}=v F, W=\eta-\tau u_{t}-\xi u_{x}$ and

$$
X=\tau(x, t, u) \frac{\partial}{\partial t}+\xi(x, t, u) \frac{\partial}{\partial x}+\eta(x, t, u) \frac{\partial}{\partial u}
$$

is any Lie point symmetry of (1.1).

The obtained conserved vector depends on $v$ because it is a conserved vector to the system

$$
\left\{\begin{array}{l}
u_{t}+\alpha u u_{x}+\beta u^{2} u_{x}+\gamma u_{x x x}+\mu u_{x x x x x}=0, \\
-v_{t}-\left(\alpha u+\beta u^{2}\right) v_{x}-\gamma v_{x x x}-\mu v_{x x x x x}=0 .
\end{array}\right.
$$

Such a conserved vector $C=\left(C^{0}, C^{1}\right)$ is, then, a nonlocal conserved vector.

\section{Local conservation laws}

According to Ibragimov's theorem on conservation laws (see also the corresponding algorithm), a conserved vector to (1.1) and (3.18) is $C=\left(C^{0}, C^{1}\right)$, whose the components are given by (4.19) and, as it was already pointed out, a nonlocal conserved vector to the original equation.

In order to find local conserved vectors, we use the Lie point symmetries found in $[18,21]$ and the fact that under the substitutions given by Theorem 3.2 , the nonlocal conserved vectors become a local conserved vector. We illustrate this fact at the same time that we proceed our calculation for establishing the fields (1.7) and (1.8).

\subsection{Conservation law for the simplified Kawahara equation}

Concerning the simplified Kawahara equation (1.5), by using the dilational symmetry

$$
X=x \frac{\partial}{\partial x}+5 t \frac{\partial}{\partial t}-4 u \frac{\partial}{\partial u}
$$


found in [18], from (4.19) we obtain

$$
\begin{aligned}
C^{0}= & -4 v u-x v u_{x}+5 \alpha t v u u_{x}+5 \mu t v u_{x x x x x}, \\
C^{1}= & x v u_{t}-4 \alpha v u^{2}-4 \mu u v_{x x x x}-\mu x u_{x} v_{x x x x}-5 \alpha t v u u_{t} \\
& -5 \mu t u_{t} v_{x x x x}+5 \mu u_{x} v_{x x x}+\mu x u_{x x} v_{x x x}+5 \mu t u_{x t} v_{x x x} \\
& -6 \mu v_{x x} u_{x x}-\mu x v_{x x} u_{x x x}-5 \mu t v_{x x} u_{x x t}+7 \mu v_{x} u_{x x x} \\
& +\mu x v_{x} u_{x x x x}+5 \mu t v_{x} u_{x x x t}-8 \mu v u_{x x x x}-5 \mu t v u_{x x x x t} .
\end{aligned}
$$

Substituting $v=x-\alpha t u$ into the components above, we arrived at

$$
\begin{aligned}
C^{0}= & -2 x u+\alpha t u^{2}+D_{x}\left(-x^{2} u+3 \alpha x t u^{2}-\frac{5}{3} \alpha^{2} t^{2} u^{3}\right) \\
& +D_{x}\left(5 \mu x t u_{x x x x}-5 \mu t u_{x x x}-5 \alpha \mu t^{2} u u_{x x x x}+5 \alpha \mu t^{2} u_{x} u_{x x x}-\frac{5}{2} \alpha \mu t^{2} u_{x x}^{2}\right), \\
C^{1}= & -\alpha x u^{2}+\frac{2}{3} \alpha^{2} t u^{3}-2 \mu x u_{x x x x}+2 \mu u_{x x x}+2 \alpha \mu t u u_{x x x x} \\
& -2 \alpha \mu t u_{x} u_{x x x}+\alpha \mu t u_{x x}^{2}+D_{t}\left(x^{2} u-3 \alpha x t u^{2}+\frac{5}{3} \alpha^{2} t^{2} u^{3}\right) \\
& D_{t}\left(-5 \mu x t u_{x x x x}+5 \mu t u_{x x x}+5 \alpha \mu t^{2} u u_{x x x x}-5 \alpha \mu t^{2} u_{x} u_{x x x}+\frac{5}{2} \alpha \mu t^{2} u_{x x}^{2}\right)
\end{aligned}
$$

Once transferred the term $D_{x}(\cdots)$ from $C^{0}$ to $C^{1}$, we find the conserved vector $C=\left(C^{0}, C^{1}\right)$, where

$$
\begin{aligned}
C^{0}= & -2 x u+\alpha t u^{2}, \\
C^{1}= & -\alpha x u^{2}+\frac{2}{3} \alpha^{2} t u^{3}-2 \mu x u_{x x x x}+2 \mu u_{x x x}+2 \alpha \mu t u u_{x x x x} \\
& -2 \alpha \mu t u_{x} u_{x x x}+\alpha \mu t u_{x x}^{2}
\end{aligned}
$$

for the simplified Kahawara equation.

Now, substituting $v=u$ into (5.21), it is obtained

$$
\begin{aligned}
C^{0}= & -4 u^{2}-x u u_{x}+5 \alpha t u^{2} u_{x}+5 \mu t u u_{x x x x x}, \\
C^{1}= & x u u_{t}-4 \alpha u^{3}-12 \mu u u_{x x x x}-5 \alpha t u^{2} u_{t}-5 \mu t u_{t} u_{x x x x}+12 \mu u_{x} u_{x x x}+ \\
& 5 \mu t u_{x t} u_{x x x}-6 \mu\left(u_{x x}\right)^{2}-5 \mu t u_{x x} u_{x x t}+5 \mu t u_{x} u_{x x x t}-5 \mu t u u_{x x x x t}
\end{aligned}
$$

TEMA Tend. Mat. Apl. Comput., 14, No. 1 (2013), 109-118.

doi: 10.5540/tema.2013.014.01.0109 
and then,

$$
\begin{aligned}
C^{0}= & -\frac{7}{2} u^{2}+D_{x}\left(-\frac{x u^{2}}{2}+\frac{5}{3} \alpha t u^{3}+5 \mu t u u_{x x x x}-5 \mu t u_{x} u_{x x x}+\frac{5}{2} \mu t u_{x x}^{2}\right), \\
C^{1}= & -\frac{7}{3} \alpha u^{3}-7 \mu u u_{x x x x}+7 \mu u_{x} u_{x x x}-\frac{7}{2} \mu u_{x x}^{2} \\
& -D_{t}\left(-\frac{x u^{2}}{2}+\frac{5}{3} \alpha t u^{3}+5 \mu t u u_{x x x x}-5 \mu t u_{x} u_{x x x}+\frac{5}{2} \mu t u_{x x}^{2}\right) .
\end{aligned}
$$

After a straightforward calculation and multiplying the final result by $-7 / 2$, it is obtained the vector (1.7).

\subsection{Conservation law for the Gardner equation}

In order to establish the conserved vector (1.8) for the Gardner equation, we first substitute the components of the following Lie point symmetry generator

$$
X_{3}=(2 x+6 t) \frac{\partial}{\partial x}+6 t \frac{\partial}{\partial t}-(2 u+1) \frac{\partial}{\partial u}
$$

into the Eq. (4.19) with $\mu=0, \alpha=\beta=6$ and $\gamma=1$. Thus

$$
\begin{aligned}
C^{0}= & -6 v t\left(6 u+6 u^{2}\right) u_{x}-6 v t u_{x x x}-(2 u+1) v-(2 x+6 t) v u_{x}, \\
C^{1}= & (2 x+6 t) v u_{t}-(2 x+6 t) v u_{x x x} \\
& +(2 u+1)\left(6 u+6 u^{2}\right) v+(2 u+1) v_{x x}+(2 x+6 t) v_{x x} u_{x} \\
& +6 t\left(6 u+6 u^{2}\right) v u_{t}+6 t v_{x x} u_{t}-4 v_{x} u_{x}-(2 x+6 t) v_{x} u_{x x} \\
& -6 t v_{x} u_{x t}+6 v u_{x x}+(2 x+6 t) v u_{x x x}+6 t v u_{x x t} .
\end{aligned}
$$

Now, setting $v=u$ into (5.22), it is obtained

$$
\begin{aligned}
C^{0}= & -36 t u^{2} u_{x}-36 t u^{3} u_{x}-6 t u u_{x x x}-2 u^{2}-u-2 x u u_{x}-6 t u u_{x}, \\
C^{1}= & 2 x u u_{t}+6 t u u_{t}+18 u^{3}+12 u^{4}+6 u^{2}+2 u u_{x x}+u_{x x}+36 t u^{2} u_{t} \\
& +36 t u^{3} u_{t}+6 t u_{t} u_{x x}-4\left(u_{x}\right)^{2}-6 t u_{x} u_{x t}+6 u u_{x x}+6 t u u_{x x t},
\end{aligned}
$$

which are equivalent to

$$
\begin{aligned}
C^{0}= & -u-u^{2}-D_{x}\left(x u^{2}+3 t u^{2}+6 t u u_{x x}-3 t\left(u_{x}\right)^{2}+9 t u^{4}+12 t u^{3}\right), \\
C^{1}= & 3 u^{4}+6 u^{3}+3 u^{2}+2 u u_{x x}+u_{x x}-\left(u_{x}\right)^{2} \\
& +D_{t}\left(x u^{2}+3 t u^{2}+6 t u u_{x x}-3 t\left(u_{x}\right)^{2}+9 t u^{4}+12 t u^{3}\right) .
\end{aligned}
$$

Then, after reckoning, we get the vector field (1.8). 


\section{Conclusion}

In this review paper we have revisited some recent results connecting Lie symmetries and conservation laws for equations not necessarily being from Euler-Lagrange equations. Moreover, we have illustrated our discussion with some new conservation laws obtained via Ibragimov's approach. Such conservation laws are established, at least using such a new development, for the first time in the present review.

The interested reader can found more local conservation laws for equations of the type (1.1), where we established conservation laws for the general Kawahara equation (1.3) and modified Kawahara equation (1.5). Moreover, some conservation laws for the $\mathrm{KdV}$ equation can also be established using those obtained results, see also [16]. More recently, some new classes of evolution equations up to fifth order have been discovered, see [7].

Resumo. Neste trabalho revisitamos alguns resultados recentes sobre leis de conservação de uma classe de equações evolutivas até quinta ordem.

Palavras-chave. Teorema de Ibragimov, leis de conservação, equações KdV de quinta ordem.

\section{References}

[1] G.W. Bluman, S. Kumei, "Symmetries and Differential Equations", Applied Mathematical Sciences 81, Springer, New York, 1989.

[2] I.L. Freire, Conservation laws for self-adjoint first order evolution equations, $J$. Nonlin. Math. Phys., 18 (2011), 279-290.

[3] I.L. Freire, Self-adjoint sub-classes of third and fourth-order evolution equations, Appl. Math. Comp., 217 (2011), 9467-9473.

[4] I.L. Freire, New conservation laws for inviscid Burgers equation, Comp. Appl. Math., 31 (2012), 559-567.

[5] I.L. Freire, J. C. S. Sampaio, Nonlinear self-adjointness of a generalized fifthorder KdV equation, J. Phys. A: Math. Theor., 45 (2012), 032001.

[6] I.L. Freire, J.C.S. Sampaio, Conservation laws for Kawahara equations, Anais do XXXIV CNMAC, 2012.

[7] I.L. Freire, New classes of nonlinearly self-adjoint evolution equations of thirdand fifth-order, Commun. Nonlinear Sci. Numer. Simulat., 18 (2013), 493-499.

[8] M.L. Gandarias, Weak self-adjoint differential equations, J. Phys. A, 44 (2011), 262001.

[9] M.L. Gandarias, Weak self-adjointness and conservation laws for a porous medium equation, Commun. Nonlinear Sci. Num. Sci., 17 (2012), 2342-2349. 
[10] M.L. Gandarias, M. Redondo, M.S. Bruzón, Some weak self-adjoint HamiltonJacobi-Bellman equations arising in financial mathematics, Nonlin. Anal. RWA, 13 (2012), 340-347.

[11] M.L. Gandarias, M. S. Bruzón, Some conservation laws for a forced KdV equation, Nonlin. Anal. RWA, 13 (2012), 2692-2700.

[12] N.H. Ibragimov, A new conservation theorem, J. Math. Anal. Appl., 333 (2007), 311-328.

[13] N.H. Ibragimov, M. Torrisi, R. Tracinà, Quasi self-adjoint nonlinear wave equations, J. Phys. A: Math. Theor., 43 (2010), 442001-442009.

[14] N.H. Ibragimov, M. Torrisi, R. Tracinà, Self-adjointness and conservation laws of a generalized Burgers equation, J. Phys. A: Math. Theor., 44 (2011), 145201145206 .

[15] N.H. Ibragimov, Nonlinear self-adjointness and conservation laws, J. Phys. A: Math. Theor., 44 (2011), 432002-432006.

[16] N.H. Ibragimov, Nonlinear self-adjointness in constructing conservation laws, Archives of ALGA, 7/8 (2011), 1-90.

[17] N.H. Ibragimov, "Transformation groups applied to mathematical physics", Translated from the Russian Mathematics and its Applications (Soviet Series), D. Reidel Publishing Co., Dordrecht, 1985.

[18] H. Liu, J. Li, L. Liu, Lie symmetry analysis, optimal systems and exact solutions to the fifth-order KdV types of equations, J. Math. Anal. Appl., 368 (2010), 551-558.

[19] R. Naz, F.M. Mahomed, D.P. Mason, Comparison of different approaches to conservation laws for some partial differential equations in fluid mechanics, Appl. Math. Comput., 205 (2008), 212-230.

[20] P.J. Olver, "Applications of Lie groups to differential equations", Springer, New York, (1986).

[21] J.C.S. Sampaio, "Simetrias de Lie e leis de conservação de equações evolutivas do tipo Korteweg-de Vries', Dissertação de Mestrado, Programa de PósGraduação em Matemática Aplicada, UFABC, Santo André-SP, 2012.

[22] M. Torrisi, R. Tracinà, Quasi self-adjointness of a class of third order nonlinear dispersive equations, Nonlin. Anal.: RWA, 14 (2013), 1496-1502. 